\title{
Entscheidung und Kommunikation im Heilberuf
}

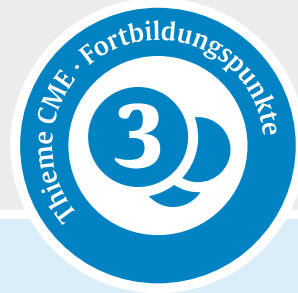

Klaus Spremann¹, Georg Adler², Johannes Treib ${ }^{3}$

1 Universität St. Gallen

2 Institut für Studien zur Psychischen Gesundheit (ISPG), Mannheim

3 Klinik für Neurologie, Westpfalz-Klinikum, Kaiserslautern

\section{ZUSAMMENFASSUNG}

Der Arzt muss seine medizinische Kompetenz und Leistungen so weit sehen, dass sie diversen kommunikativen Anforderungen genügt. Voran stehen die Aufklärung des Patienten und die Prüfung der Einwilligungsfähigkeit. Verweigert ein (einwilligungsfähiger) Patient die Zustimmung (Non-Konkordanz), geht er von offenbar anderen Einschätzungen aus, die sich meist als nicht korrekte Eigenbeurteilung, Betonung eigener Vorstellungen oder Selbstüberschätzung herausstellen. Dann zeigt der Dunning-Kruger-Effekt einen Weg zur Überwindung: Der Arzt muss Reflexion einfordern und dazu das Patientengespräch in Komplexität und Tiefe ausweiten. Schließlich können sich Mängel in der Therapietreue zeigen. Je nach Ursache der Non-Adhärenz muss der Arzt im nicht direktiven Gespräch versuchen, die Einzelschritte an Patientengewohnheiten zu knüpfen oder im direktiven Gespräch Bedenken ausräumen und an das Therapieziel erinnern.

\section{Grundlagen}

\section{Einwilligungsfähigkeit}

Der Patient entscheidet: Bevor eine Heilbehandlung oder Therapie beginnt, muss der Patient - oder an seiner Stelle ein Betreuer - Einverständnis gegeben haben. Arzt oder Therapeut dürfen die Einwilligung eines Patienten aber nur annehmen, wenn dieser fähig ist, seine Situation sowie die Bedeutung, Tragweite und die Risiken der ärztlichen Maßnahmen zu erfassen und darauf aufbauend seinen Willen bilden kann (Einwilligungsfähigkeit §1901a BGB). Das ist eine starke Vorbedingung. Ist ein Patient einwilligungsfähig, dürfen keine medizinischen Maßnahmen gegen seinen Willen vorgenommen werden. Ist ein Patient zwar mit den ärztlichen Maßnahmen einverstanden, besitzt aber keine Einwilligungsfähigkeit, dann würde der Arzt mit seinen Maßnahmen eine Straftat begehen (auch wenn diese straffrei bliebe, weil sich der Patient bereit erklärte). Ist Einwilligungsfähigkeit nicht gegeben, greift das Betreuungsrecht [5].

Die Einwilligungsfähigkeit verlangt zunächst Kenntnis und Verständnis. Der Patient muss den Sachverhalt, die in Frage kommenden Maßnahmen sowie Folgen und Risiken kennen und verstehen. Sodann muss der Patient all diese Informationen verarbeiten, mit anderem Wissen verknüpfen, bewerten und zu diesem Zweck abwägen und vergleichen können. Und er muss in der Lage sein, auf der Grundlage von Kenntnis, Verständnis, Verarbeitung und Bewertung seinen (wohlüberlegten) Willen bestimmen zu können.
Um die rechtlich wirksame Annahme eines Therapievorschlags zu erhalten, müssen Arzt oder Therapeut 2 Vorarbeiten erbringen. Eine ist die Aufklärung des Patienten: Der Arzt bietet allgemeine und patientenspezifische Informationen über die Krankheit, erläutert einen von ihm vorgeschlagenen Therapieweg, nennt Alternativen und spricht an, welche Mitwirkung des Patienten üblich ist oder erwartet wird. Eine zweite Vorarbeit ist die Prüfung der Einwilligungsfähigkeit. Dies kann durch Fragen geschehen, deren Beantwortung dem Arzt zeigt, dass der Patient die mit der Aufklärung gebotenen Informationen verstanden hat, weiterverarbeiten kann, Einsicht in Vorteile und Risiken gewonnen hat, diese abwägen und für sich bewerten kann und, eventuell im Vergleich mit Alternativen, eine Erwartung für den persönlichen Nutzen der vorgeschlagenen ärztlichen Maßnahme bilden kann. Oft zeigt bereits die Interaktion im Aufklärungsgespräch, ob Verständnis und Urteilsvermögen vorhanden sind. Dann müssen keine Fragen zusätzlich gestellt werden, um die Einwilligungsfähigkeit zu prüfen.

\section{Konkordanz und Therapieadhärenz}

Ist die Aufklärung erfolgt, Einwilligungsfähigkeit gegeben und stimmt der Patient dem Therapievorschlag zu, dann liegt Konkordanz vor: Die ärztliche Sicht einerseits und die Einsicht und die wohlüberlegte Willensbildung des Patienten andererseits kommen zur Übereinstimmung. Die Therapie kann daraufhin begonnen werden. Sollte sich der Patient zwar als einwilligungsfähig erweisen, gelangt aber zu einer Ablehnung der Therapie (Non-Konkordanz), dürfte der Arzt oder Therapeut bemüht sein, weitere Informationen zu bieten. In den nachfolgenden Abschnitten soll geklärt werden, welche Art von Kommunikation geeignet 
sein kann, einen einwilligungsfähigen Patienten, der ablehnt, doch noch zu überzeugen.

Unterstützt der Patient sodann die Behandlungen und Maßnahmen wie erwartet pünktlich, genau und dauerhaft, dann wird von Therapietreue oder Therapieadhärenz gesprochen. Mit Therapieadhärenz wird das Ausmaß bezeichnet, in dem sich der Patient an die getroffene Vereinbarung hält und persistent mitwirkt. Ist sein Verhalten in dieser Hinsicht mangelhaft, liegt Non-Adhärenz vor. Leider kommt es im Verlauf einer Heilbehandlung oft - die WHO berichtet über $50 \%$ der Fälle - dazu, dass der Patient nicht so mitwirkt, wie es üblich ist oder vereinbart wurde: Non-Adhärenz tritt ein. Dann kommen dem Arzt oder Therapeuten als weitere informatorische Aufgabe zu, den Patienten dazu zu verhelfen oder dazu zu bringen, die Therapie wie vereinbart zu unterstützen. Welche Art der Kommunikation dazu geeignet ist, wird gleich anschließend (aus der Literatur) rekapituliert.

Der Komplex des Patientenverhaltens, der sich auf Einsicht, Einverständnis, Bereitwilligkeit und Mitwirkung bezieht, wurde früher als „Compliance“ bezeichnet. Damit wurde der Grad der Einhaltung von (ärztlichen) Vorgaben und (medizinischen) Regeln bezeichnet. Heute werden die Patientenwürde, die Selbstbestimmung des Patienten und die körperliche Integrität als Grundrechte betont. Selbst wenn das Betreuungsgesetz greift, muss das Wohl des Betreuten vorrangig durch ihn selbst bestimmt werden. Deshalb ist die stigmatisierende Begrifflichkeit der Compliance durch die beiden Begriffe der Konkordanz sowie der Therapieadhärenz ersetzt.

\section{Kommunikation bei Non-Adhärenz}

Bei Non-Adhärenz stehen 2 Situationen im Vordergrund: Erstens kann sich der Patient als nachlässig oder willensschwach erweisen. Zum Beispiel vergisst er die Einnahme von Medikamenten, versäumt Termine, schafft es nicht, seinen Lebensstil zu ändern. Die Situation kann durch das Matthäus-Wort beschrieben werden, dass der Geist willig, aber das Fleisch schwach ist. Als Ursache für diese Form von Non-Adhärenz wird zu geringes Selbstvertrauen oder das Fehlen von Orientierungspunkten für das Verhalten gesehen. Um dem Patienten Kraft für die Einhaltung des Therapiewegs zu geben, versucht der medizinische Betreuer im Gespräch, das Selbstvertrauen des Patienten stärken. Zusätzlich werden die für die Therapie verlangten Einzelaktionen des Patienten an seine Gewohnheiten gebunden. Die Stärkung des Selbstvertrauens und die genannten Planungshilfen setzen eine nicht direktive Kommunikation voraus [24].

Die zweite Situation von Non-Adhärenz ist durch Labilität, Ängste und Befürchtungen charakterisiert. Der Patient stellt das gegebene Einverständnis immer wieder infrage. Die Forschung empfiehlt dann die motivierende Gesprächsführung (Motivational Interviewing MI) nach
Miller/Rollnick [18]. Im gezielten Gespräch erkundet der Therapeut zunächst die Bedenken und macht klar, weshalb sie keine große Relevanz haben. Sodann erinnert er an die Ziele der Therapie. Bei MI führt der Berater das Gespräch zwar partnerschaftlich, verzichtet mithin auf konfrontatives Vorgehen. Indes wendet er eine direktive Intervention an. So wird die intrinsische Motivation des Patienten geweckt, die Umsetzungskompetenz verbessert und die Handlungsbereitschaft gestärkt ( Tab. 1).

\section{Ablehnung des Therapievorschlags}

\section{Objektive versus subjektive Bewertung}

Ein einwilligungsfähiger Patient könnte die von Arztseite vorgeschlagene Therapie ablehnen. Kommt der Patient wohlüberlegt zu einer Absage, wird das zunächst überraschen. Denn der Arzt lässt bei seinen Untersuchungen und Vorschlägen Vernunft walten: Er folgt der Wissenschaft, hält sich an Best Practices und berücksichtigt die Besonderheiten des Einzelfalls. Und für den Patienten ist die Einwilligungsfähigkeit ebenso Vernunft. Denn Vernunft bedeutet die „geistige Fähigkeit des Menschen, Einsichten zu gewinnen, sich ein Urteil zu bilden, Zusammenhänge und Ordnung des Wahrgenommenen zu erkennen und sich danach zu richten“ (Oxford Lexikon). Genau diese Qualitäten definieren die Einwilligungsfähigkeit. Weil sowohl der Arzt als auch der einwilligungsfähige Patient vernünftig vorgehen, sollten sie zu übereinstimmenden Schlussfolgerungen gelangen.

Von daher kann eine ausbleibende Konkordanz - der einwilligungsbefähigte Patient lehnt den Therapievorschlag ab - nur durch Unterschiede in den Ausgangsinformationen erklärt werden, die zwischen dem vernünftigen Vorschlag des Arztes und der vernünftigen Willensbildung des Patienten bestehen. Beispielsweise geht der Arzt von einem Patienten mit normaler Geduld aus, während der Patient seine eigene große Ungeduld ins Kalkül zieht. Oder der Arzt schätzt die Wahrscheinlichkeiten für Nebenwirkungen aufgrund der statistischen Häufigkeit ein, während der Patient bei einer Eintrittswahrscheinlichkeit von $10 \%$ bereits mit einem Ereignis rechnet, das ihn mit Sicherheit treffen wird.

Bei Unterschieden in den Ausgangsinformationen der beiden Seiten wird es sich also um die Gewichtung handeln, mit der die Besonderheiten der konkreten Situation einfließen, wie die Präferenzen des Patienten und die Einschätzungen der Wahrscheinlichkeiten. In der Tat wird der Arzt die Besonderheiten der Situation und Einstellungen des Patienten angemessen berücksichtigen, unter Beachtung objektivierter empirischer Daten und „typischer“ Einschätzungen. Der Patient hingegen wird seiner eigenen Sicht folgen, persönliche Erlebnisse betonen, eigenen Präferenzen und eigene Wahrscheinlichkeitseinschätzungen als gegeben betrachten. Dann wird er daraus mit Verstand und 
- Tab. 1 Der Arzt oder Therapeut muss in Gesprächen aufklären, die Entscheidungsfähigkeit des Patienten prüfen und bei Non-Adhärenz geeignete Hilfen bieten

\begin{tabular}{|c|c|c|}
\hline Gespräch & Inhalt & Zweck \\
\hline 1. Aufklärung & $\begin{array}{l}\text { 1. Welche generellen Fakten und und welche patientenspezifi- } \\
\text { schen Informationen sollen vermittelt werden? } \\
\text { 2. Welche Therapie wird vorgeschlagen und welche Alternativen } \\
\text { werden genannt? }\end{array}$ & $\begin{array}{l}\text { Schaffung der informatorischen Basis für die } \\
\text { Patientenentscheidung und Vorbereitung einer } \\
\text { Therapievereinbarung. }\end{array}$ \\
\hline $\begin{array}{l}\text { 2. Prüfung der Einwilligungs- } \\
\text { fähigkeit }\end{array}$ & $\begin{array}{l}\text { 1. Was sagt der Ablauf der Gespräche über die Einsicht und } \\
\text { Urteilsfähigkeit des Patienten aus? } \\
\text { 2. Mit welchen zusätzlichen Fragen kann geklärt werden, wie gut } \\
\text { der Patient die Ziele und Risiken einschätzen und bewerten kann? }\end{array}$ & $\begin{array}{l}\text { Erfüllung der gesetzlichen Pflicht, einer Bejahung } \\
\text { des Therapievorschlags nur dann zu akzeptieren, } \\
\text { wenn der Patient fähig ist, seine Einwilligung zu } \\
\text { geben. }\end{array}$ \\
\hline $\begin{array}{l}\text { 3. Hilfe bei Non-Ahärenz } \\
\text {... in Form von Nachlässigkeit }\end{array}$ & $\begin{array}{l}\text { 1. Ermutigung und Stärkung des Selbstwerts. } \\
\text { 2. Therapieschritte planen und dazu mit Lebensgewohnheiten } \\
\text { koppeln. }\end{array}$ & $\begin{array}{l}\text { Die Stärkung des Selbstwerts des Patienten und } \\
\text { Planungshilfen sollen die genaue und dauerhafte } \\
\text { Mitwirkung des Patienten unterstützen. }\end{array}$ \\
\hline ... in Form von Labilität & $\begin{array}{l}\text { 1. Mit motivierender Gesprächsführung die Bedenken erkunden } \\
\text { und klare Aussagen zu deren Relevanz treffen. } \\
\text { 2. Die Therapieziele in Erinnerung rufen. }\end{array}$ & $\begin{array}{l}\text { Der Patient soll seine intrinsische Motivation } \\
\text { wiederbeleben und seine Umsetzungskompetenz } \\
\text { stärken. }\end{array}$ \\
\hline
\end{tabular}

Logik Schlüsse ziehen und womöglich die empfohlenen Maßnahmen ablehnen.

Der Arzt basiert einen mit Vernunft formulierten Vorschlag unter angemessener Berücksichtigung der Besonderheiten des Patienten, während der Patient seine subjektiven Vorstellungen überbetonen könnte, bevor er - zugegeben mit Logik und Verstand - zu seiner persönlichen Entscheidung gelangt. Dem Arzt darf zuerkannt werden, dass er offen ist gegenüber allen Informationen der medizinischen Wissenschaft und Praxis, während der Patient seine eigenen Erfahrungen und Einschätzungen stärker berücksichtigen dürfte. Eine Folge ist, dass der Arzt seine Sicht und Empfehlung, auch die angemessene Berücksichtigung der Besonderheiten des Patienten, einem sachverständigen Dritten erklären kann, der sie dann ebenso sachgerecht und objektiv ansieht. Der Patient hingegen findet bei anderen für seine Entscheidung womöglich kein Gehör, weil sie seine persönlichen Einschätzungen und Erfahrungen als zu subjektiv nicht teilen.

\section{Überwindung der Non-Konkordanz}

Wenn im Gespräch deutlich wird, dass ein Patient zwar entscheidungsfähig ist, also die Lage erfassen kann und zu logischen Schlussfolgerungen bereit ist, dass er aber von einer stark auf das Selbst (eigene Erfahrungen, eigene Präferenzen, eigene Wahrscheinlichkeitseinschätzungen) bezogenen Sicht ausgeht, dann bestehen 2 Möglichkeiten.

Die eine ist, die zu vermutende Ablehnung des Therapievorschlags hinzunehmen. Wenn es eine Variante oder Alternative gibt, könnten Arzt oder Therapeut sie noch als Ersatz anbieten. Wenn jedoch mit der Ablehnung des Therapievorschlags die Arzt-Patientenbeziehung ausklingt, sollte dem Patienten noch gesagt werden, dass die vor- geschlagene Therapie nicht vom behandelnden Arzt erfunden wurde, sondern in langer wissenschaftlicher Arbeit mit dem Ziel gereift ist, den Menschen zu helfen. Die andere Möglichkeit besteht darin, die Enge und Selbstbezogenheit des Patienten aufzubrechen, sodass dieser auch andere Positionen und Erfahrungen zulässt und sich anderen Einstellungen gegenüber öffnet.

Um diesen Weg zu begründen, soll den Überlegungen des Patienten nachgegangen werden. Für seine Entscheidung über den Therapievorschlag, bildet der Patient Erwartungen und stellt seine Zeit- und die Risikopräferenz auf. Das Entscheidungsmodell führt auf dieses Zwischenergebnis: Patienten akzeptieren eher den Therapievorschlag, wenn sie aufgeschlossen, geduldig und sicherheitsbewusst sind. Sie neigen zur Ablehnung der Therapie, wenn sie egozentrisch, ungeduldig und risikobereit sind.

Die Verhaltensforschung identifiziert hinter diesen 3 Bestimmungsfaktoren einen Hauptfaktor, den Selbstbezug des Patienten. Nach Dunning/Kruger sind Selbstbezug und Wissensniveau negativ korreliert: Wer mehr über eine Sache weiß, neigt weniger zur Selbstüberschätzung [13] in dem Bereich. Der Dunning-Kruger-Effekt bietet daher eine Möglichkeit, die Selbstbezogenheit eines Patienten auf das gesunde Maß von Selbstwertgefühl zurückzubilden. Dazu sind die Verbesserung des eigenen Wissens und die Öffnung gegenüber anderen Argumenten notwendig. So würde das Festhalten an eigenen Vorstellungen gelöst, eine korrekte Eigenbeurteilung ermöglicht und damit der Weg zur Therapiebejahung frei. Zur Überwindung der Non-Konkordanz müssen also Wissen und Können des Betreffenden auf dem Gebiet der Krankheit und zusammenhängende Themen gesundheitlicher Lebensführung gestärkt werden. 
Dies verlangt von ärztlicher Seite, die Aufklärung auszuweiten, das Patientengespräch zu intensivieren, Reflexion zu verlangen und dabei in Komplexität, Tiefe und Querverbindungen fortzuschreiten. Zweifellos fordern diese kommunikativen Maßnahmen den Patienten, der sich bis dahin eher abgegrenzt hat. Damit er an dieser Forderung nicht zerbricht, empfiehlt sich die Stärkung psychischer Elastizität, der Resilienz. Für den Stressabbau und die Stärkung beweglicher Widerstandsfähigkeit bieten sich Gruppenübungen an [15].

\section{Struktur der Entscheidung über die Therapie}

\section{Versicherungsmodell}

Heilung bedeutet (bei schwereren Krankheiten) nicht, dass der ursprüngliche Zustand voller Gesundheit in allen Merkmalen wieder hergestellt wird. Beim kurativen Ziel ist eine neue körperliche und seelische Integrität angestrebt. Genesung (vغ̇oนaı, neomai) heißt, aus einer Gefahrensituation davon zu kommen. Patienten verstehen, dass die ärztlichen Maßnahmen beabsichtigen, eine Aggravation abzuwenden. Unbehandelt kommt es etwa bei Multipler Sklerose später häufiger zu einer progredienten Verschlechterung. Bei einem Karzinom können sich Metastasen bilden. Bei Schlaganfällen können Behinderungen Pflege verlangen. Mit rechtzeitiger Therapie wird angestrebt, die Aggravation zu verhindern. Doch die Therapie, etwa eine OP, würde schwächen und hätte wohl Nebenwirkungen. Somit sieht sich der Patient in einer Entscheidungssituation, deren Struktur dem klassischen Versicherungsproblem entspricht: Eine Person ist in eine gefährliche Lage gekommen, in der sie einen Schaden erleiden könnte. Zwar tritt der Schaden nur mit gewisser Wahrscheinlichkeit ein, und dies erst zu einem späteren Zeitpunkt. Eine Absicherung (hier: Verhinderung einer Aggravation) wäre möglich, doch damit sind Kosten verbunden (hier: durch Schwächung und Nebenwirkungen, die wohl mit der Therapie einhergehen). Sollte sich der Patient absichern oder nicht? Es handelt sich um eine Entscheidung unter Risiko. Wie der Betroffene wählt, hängt von 3 Einflussgrößen ab:

- Erwartungsbildung: Welche Informationen nimmt der Betroffene auf und welches Bild macht er sich von seiner Situation, insbesondere von den Konsequenzen seiner Wahl?

- Diskontierung: In wieweit verringert sich in der persönlichen Einschätzung die heutige Bedeutung einer Aggravation deshalb, weil es zu ihr allenfalls zu einem späteren Zeitpunkt kommen würde?

- Risikopräferenz: Wie ist die Unsicherheit und damit verbunden die geringere Planbarkeit des Lebens einzuschätzen, weil eine Aggravation nur mit einer gewissen Wahrscheinlichkeit eintritt?

\section{Rationalität versus Behaviorismus}

In der Entscheidungstheorie wird meist unterstellt, dass der Entscheidungsträger rational vorgeht. Das würde Offenheit verlangen, Aufgeschlossenheit für wissenschaftliche Erkenntnisse und Logik bei der Erwartungsbildung. Doch wir Menschen sind nur beschränkt rational und haben diverse Verzerrungen in unserem Verhalten. Eine Person könnte eigenen Vorstellungen und Vorurteilen mehr vertrauen als der Wissenschaft. Und sie könnte dem, was sie im Bekanntenkreis hört oder was die Produktwerbung im Internet verspricht, mehr Glauben schenken als Fachleuten, die auf Leitlinien, Standards und Statistiken verweisen. In der Entscheidungstheorie wird die vernunftbetonte Aufbereitung von Informationen als rationale Erwartung bezeichnet [9], wogegen im Behaviorismus das tatsächliche Vorgehen als adaptive Erwartung erkannt wird: Bei adaptiven Erwartungen geht die Person von eigenen Vorinformationen und Vorstellungen aus und ergänzt diese Schritt für Schritt durch selektierte Hinweise aus dem sozialen Umfeld [3]. Natürlich sind Mischungen zwischen rationalen und adaptiven Erwartungen zu beobachten.

Die Zeitpräferenz des Patienten bestimmt, welches heutige Gewicht er einer eventuellen Aggravation deshalb beimisst, weil sie, wenn überhaupt, erst später eintreten sollte. Wieder könnte sich ein Patient bemühen, rational vorzugehen, was auf die geläufige exponentielle Diskontierung hinausläuft [7]. Doch die Empirie zeigt, dass die meisten Menschen eine als hyperbolische Diskontierung bezeichnete Zeitpräferenz haben [2]. Sie sind anfangs ungeduldig. Der Zeithorizont ist auf den Augenblick eingeengt: Was danach passiert, hat geringe Bedeutung [1]. So erscheint eine spätere Aggravation in „weiter Ferne“ zu liegen, ganz im Gegensatz zu den Nebenwirkungen der Therapie. Ist beim Patienten der Anteil der rationalen Zeitpräferenz gering und die behavioristischen Zeitgewichtung hoch, dann dürfte er folglich eine medizinisch-wissenschaftliche Therapie eher ablehnen. Wiederum sind in der Realität Mischungen zwischen rationaler und behavioristischer Zeitpräferenz zu beobachten.

Die Risikopräferenz einer Person bestimmt, welcher Nutzen verloren geht, weil die Konsequenzen einer Wahlhandlung unsicher sind. Niemand liebt Überraschungen, die alles durcheinanderbringen. Die meisten Menschen sind risikoscheu und wollen Risiken absichern, selbst wenn sie dafür bezahlen müssen. Doch es gibt auch risikotolerante Menschen. Sie würden sich nur dann absichern wollen, wenn Absicherung günstig zu haben wäre. Ein risikoaverser Patient wählt die Therapie (trotz der Nebenwirkungen), um auf diese Weise gegen die Unsicherheit einer eventuellen Aggravation geschützt zu sein. Ein risikobereiter Patient erträgt leichter die Unsicherheit, dass niemand genau sagen kann, ob es überhaupt zu einer Aggravation kommt oder nicht. 


\section{Prospekt-Theorie}

Zur Frage, ob Menschen risikoavers oder risikobereit sind, haben sich 2 Perspektiven herausgebildet. Die erste Perspektive verfolgt Rationalität als Leitbild und postuliert das Kriterium des Erwartungsnutzens. Doch in vielen Situationen führt uns der durch die Evolution geprägte Denkprozess zu anderen Entscheidungen. Diese könnten in Urzeiten überlegen gewesen sein, als es um das Überleben ging und die Errungenschaften der modernen Zivilisation nicht zur Verfügung standen [16]. Die Verhaltenswissenschaft folgt dem Kriterium der Prospekt-Theorie. Die Prospekt-Theorie wurde durch Arbeiten von Kahneman/Tversky zum wichtigsten Paradigma des Behaviorismus [10]. Der Unterschied zwischen der rationalen und der behavioristischen Evaluation unsicherer Handlungskonsequenzen betrifft einen unsicheren Verlust oder Schaden [4]. Beim mentalen Vergleich der Handlungsmöglichkeiten wird der Eventualverlust beim Erwartungsnutzen-Kriterium stärker berücksichtigt als bei der Prospekttheorie [24]. Der Eventualverlust wird in eine vernunftorientierte Entscheidungsfindung hinsichtlich Abträglichkeit und Wahrscheinlichkeit korrekt einbezogen. Bei einer behavioristischen Entscheidungsfindung wird ein Eventualverlust hingegen quasi ausgeklammert [25]. Nach der Prospekt-Theorie wird der Patient eine Aggravation folglich als weniger abträglich (und womöglich auch als weniger wahrscheinlich) einschätzen. In der mentalen Abwägung mit den Nebenwirkungen medizinischer Behandlung fällt die Wahl des Patienten dann tendenziell gegen die Therapie aus.

\section{Overconfidence}

Die Wahl des Patienten zwischen Annahme oder Ablehnung eines medizinisch-wissenschaftlichen Therapievorschlags hängt von der persönlichen Erwartungsbildung, der Zeitpräferenz und der Risikopräferenz ab. Für jeden dieser Einflussfaktoren besteht ein Spektrum von Verhaltensweisen zwischen Vernunft (rationale Erwartungen, exponentielle Diskontierung, Erwartungsnutzen) und behavioristischer Verzerrung (adaptive Erwartungen, hyperbolische Diskontierung, Prospekt-Theorie) [23]. Da alle Spektren von einer rationalen und einer behavioristischen Verhaltensweise aufgespannt sind, besteht ein Zusammenhang. Denn eine Person ist von ihrer Grundeinstellung her eher rational orientiert oder sie handelt behavioristisch verzerrt. Diese Grundorientierung zeigt sich dann gleichermaßen im Informationsverhalten, in der Zeitpräferenz und in der Risikopräferenz. Es muss für einen Patienten also nur bestimmt werden, zu welchem Teil die Grundorientierung rational und zu welchem Teil sie behavioristisch ist.

Die Verhaltenswissenschaft zeigt, dass hinter den verschiedenen, korrelierten behavioristischen Verzerrungen ein gemeinsamer Faktor steht: die Selbstbezogenheit, Overconfidence, Hybris. Mehr oder minder ausgeprägt neigen alle Menschen zur Überschätzung ihrer (körperlichen, geistigen und seelischen) Leistungen im Vergleich zur Realität.
Sie neigen zu einer Überbewertung eigener Fähigkeiten in Relation zu den Fähigkeiten anderer Menschen. Und sie haben eine übermäßige Gewissheit hinsichtlich der Richtigkeit und Treffsicherheit eigener Urteile. Bei einer Überschätzung geht es primär nicht darum, ob die Person über viel Wissen, Kenntnisse und Fähigkeiten verfügt. Der Begriff bezieht sich auf Defizite in der Beurteilungsfähigkeit eigener und fremder Leistungen [21].

\section{Art der Kommunikation}

\section{Förderung korrekter Eigenbeurteilung}

Eine Person neigt zu Selbstüberschätzung in einem bestimmten Wissens- und Tätigkeitsbereich, sofern diese Fähigkeiten für sie relevant sind und sie darin eine gewisse Grundkompetenz besitzt. Für einen Patienten ist der Bereich von Gesundheit und Lebensführung relevant. Die Themenpunkte betreffen die Therapie, eine Schwächung durch Nebenwirkungen, eine eventuelle Aggravation, Konsequenzen und die Zeitdauern. Folge: Patienten, die sich darin nicht überschätzen, sind eher offen für eine Therapie. Personen, die sich darin überschätzen und eigenen Vorstellungen folgen, lehnen die Therapie oftmals ab.

Dunning/Kruger haben gezeigt, dass sich eine Person weniger überschätzt, wenn sie in dem betreffenden Sachbereich über das Elementare hinaus mehr Können und Wissen hat: Das Niveau der Fähigkeiten in einer Sache und das Vermögen, diese Fähigkeiten realistisch beurteilen zu können, sind korreliert. Der Dunning-Kruger-Effekt besagt: Wer mehr weiß und fähiger ist, der kann Leistung, Können und Kompetenz besser beurteilen und neigt weniger zur Selbstüberschätzung [13]. Der Grad an Selbstüberschätzung ist womöglich veränderlich: Da Wissen und Können mit der Fähigkeit zur korrekten Eigenbeurteilung korreliert sind, sollte es möglich sein, Überschätzung auf ein gesundes Maß von Selbstwertgefühl zurückzubilden [19]. Ergebnis: Die Person könnte dann zu einer stärker rationalen Grundhaltung finden und als Patient die Therapie bejahen. Der Weg: Wissen und Können des Betreffenden stärken.

\section{Fordernder Dialog}

Die ärztliche Seite müsste Patientengespräche intensivieren und in der Komplexität der Inhalte von Gespräch zu Gespräch fortschreiten. Das übersteigt die zeitlichen Möglichkeiten von Praxis und Klinik. Es ließe sich allenfalls in der Form bewältigen, dass einem Patienten Handreichungen und Lesehinweise für das Selbststudium gegeben werden und deren Reflexion zu verlangen. Die Ärzte müssten die Weiterbildung des Patienten auf dem Gebiet seiner Krankheit einfordern.

Das Niveau des Dialogs soll also zunehmend anspruchsvoller werden. Verständlichkeit ja, Unterforderung nein. Geistige Forderung, Übung und Bildung reduzieren den Selbstbezug bis auf ein gesundes Selbstwertgefühl. Der 
Patient wird fähig, eigene und fremde Gedanken angemessen wahrzunehmen und abzuwägen. Dieser Weg zur Verbesserung intentionaler Non-Adhärenz stellt eine Forderung dar, weil sich ein bis dahin abgrenzender Patient öffnen und anderes zulassen soll. An dieser Forderung darf der Patient nicht zerbrechen. Auf dem Weg der Öffnung zu mehr Wissen und Können muss der Patient deshalb seine Resilienz stärken, die psychische Elastizität [15]. Sie zeigt sich als Fähigkeit, aus dem Widerspruch zwischen eigenen Vorstellungen und geforderter Rationalität die Kraft für eigene Weiterentwicklung zu schöpfen. Dazu sollten die Gesprächspartner auf der ärztlichen Seite Chancen erkennen lassen und zu Übungen anregen, die Gelassenheit geben. Entspannung und Ruhe sind förderlich, die Resilienz zu stärken [20].

Der Weg zu Rationalität und Resilienz verlangt Übung und Ausbildung durch Reflexion, Dialog und Interaktion mit anderen Menschen. Parallel muss die seelische Beweglichkeit des Patienten gestärkt werden, Brücken zwischen außen und innen schlagen zu können. Die Entwicklung eigener Fähigkeiten des Patienten und die Stärkung der Resilienz sind die Schlüssel, um Überheblichkeit in Selbstvertrauen zu wandeln. Sie sind damit ebenso der Schlüssel, um die Öffnung des Patienten zu fördern, der so zu einer positiven Haltung gegenüber dem Therapievorschlag gelangt.

\section{FAZIT}

Auch wenn Konkordanz und Therapieadhärenz Einsicht, Einverständnis, Bereitwilligkeit und Mitwirkung des Patienten beschreiben, ist ihre Verbesserung nicht allein eine Aufgabe für den Patienten, sondern ebenso für den Arzt oder Therapeuten. Dem Arzt kommt folglich neben den medizinischen Maßnahmen eine kommunikative Aufgabe mit mehreren Zielen zu, die jeweils spezielle kommunikative Ansätze verlangen.

1. Der Arzt muss zur Krankheit generelle sowie patientenspezifische Informationen bieten, seinen Therapievorschlag erläutern und gegebenenfalls Alternativen nennen. Bei der Aufklärung wird vom Arzt verlangt, sich verständlich auszudrücken.

2. Dabei muss der Arzt die Einwilligungsfähigkeit des Patienten prüfen, wozu die Fähigkeit gehört, Einsichten zu gewinnen, mit Verstand und Logik Schlüsse zu ziehen, die Situation und Risiken zu bewerten und darauf aufbauend eine überlegte Entscheidung zum Therapievorschlag zu bilden.

3. Bevor es zur Entscheidung über Annahme oder Ablehnung des Therapievorschlags kommt, sollte der Arzt einschätzen, wie offen oder selbstbezogen der Patient ist. Denn wie das Versicherungsmodell zeigt, neigen Patienten mit starker Selbstbezogenheit (Overconfidence) typischerweise zu einer Ablehnung des Therapievorschlags - dies gleichwohl mit Verstand und Logik. Der Arzt kann sein Gespräch dann tiefer und fordernder ausrichten und vom Patienten Reflexion verlangen. Denn mit einer Hinterfragung der Ausgangsinformationen des Patienten und der Wissensverbreiterung kann (aufgrund des Dunning-Kruger-Effekts) eine Öffnung begünstigt werden, durch die rein subjektive zu objektiven Ausgangsinformationen werden können.

4. Eine vierte Kommunikation ist angebracht, wenn der (einwilligungsfähige) Patient dann zustimmt, die Heilbehandlung einsetzt, jedoch eine geringe Therapietreue deutlich wird. Geht die Non-Adhärenz auf Nachlässigkeit zurück, wird der Arzt ermutigen, den Selbstwert des Patienten steigern und zeigen, wie die einzelnen Therapieschritte geplant und mit Lebensgewohnheiten gekoppelt werden können. Hängt die Non-Adhärenz hingegen mit Labilität zusammen, dann wird der Arzt mit der Technik motivierender Gesprächsführung (MI nach Rollnick) die Bedenken erkunden, klare Aussagen zu deren Relevanz treffen, und an die Therapieziele erinnern.

Interessenkonflikt

\section{Erklärung zu finanziellen Interessen}

Forschungsförderung erhalten: nein; Honorar/geldwerten Vorteil für Referententätigkeit erhalten: nein; Bezahlter Berater/interner Schulungsreferent/Gehaltsempfänger: nein; Patent/Geschäftsanteile/Aktien (Autor/Partner, Ehepartner, Kinder) an Firma (Nichtsponsor der Veranstaltung): nein; Patent/Geschäftsanteile/Aktien (Autor/Partner, Ehepartner, Kinder) an Firma (Sponsor der Veranstaltung): nein.

Erklärung zu nicht finanziellen Interessen

Die Autoren geben an, dass kein Interessenkonflikt besteht.

\section{Korrespondenzadresse}

Prof. Dr. med. Johannes Treib

Klinik für Neurologie

Westpfalz-Klinikum GmbH

Hellmut-Hartert-Str. 1, 67655 Kaiserslautern

Deutschland

Tel. 0631/2031040

jtreib@westpfalz-klinikum.de 
[1] Ainslie G. Specious Reward: A Behavioral Theory of Impulsiveness and Impulse Control. Psychological Bulletin 1975; 82 : 463-496

[2] Benzion U, Rapoport A, Yagil J. Discount Rates Inferred from Decisions: An Experimental Study. Management Science 1989; 35: 270-284

[3] Burchardt T. Are One Man's Rags Another Man's Riches? Identifying Adaptive Expectations using Panel Data. Soc Indic Res 2005; 74: 57-102

[4] Eeckhoudt L, Fiori AM, Gianin ER. Risk Aversion, Loss Aversion, and the Demand for Insurance. Risks 2018; 6: 1-19

[5] Erhard D. Die Einwilligungsfähigkeit des Patienten. Lege artis 2012; 2: 292-295

[6] Fernbach PM, Rogers T, Fox CR, et al. Political Extremism is supported by illusion of understanding. Psychological science 2013; 24: 939-946

[7] Frederick S, Loewenstein G, ODonoghue T. Time Discounting and Time Preference: A Critical Review. J Econ Literature 2002; 40: 351-401

[8] Gast A, Mathes T. Medication adherence influencing factors - an (updated) overview of systematic reviews. Syst Rev 2019; 8: 112

[9] Hielscher T. Unsicherheit, Erwartungen und die Hypothese rationaler Erwartungen. Diskussionsbeiträge des Fachbereichs Wirtschaftswissenschaft der Freien Universität Berlin. Berlin: Volkswirtschaftliche Reihe 1999; 18

[10] Kahneman D, Tversky A. Prospect Theory - An analysis of decision under risk. Econometrica 1979; 47: 263-291

[11] Kardas P, Lewek P, Matyjaszczyk M. Determinants of patient adherence: a review of systematic reviews. Front Pharmacol 2013; 4: 91

[12] Krueger KP et al. Medication adherence and persistence: a comprehensive review. Adv Ther 2005; 22(4): 313-356

[13] Kruger ], Dunning D. Unskilled and unaware of it. How difficulties in recognizing one's own incompetence lead to inflated self-assessments. Journal of Personality and Social Psychology 1999; 77: 1121-1134

[14] Krueger KP et al. Medication adherence and persistence: a comprehensive review. Adv Ther 2005; 22(4): 313-356

[15] Leipold B, Greve W. Resilience: A conceptual bridge between coping and development. European Psychologist 2009; 14 : $40-50$
[16] McDermott R, Fowler JH, Smirnov O. On the Evolutionary Origin of Prospect Theory Preferences. Journal of Politics 2008; 70: 335-350

[17] Miller WR, Rollnick S. Motivational interviewing: Preparing people to change addictive behavior. Miller WR, Rollnick S. Motivierende Gesprächsführung - Motivational Interviewing. Freiburg im Breisgau: Lambertus, 2005

[18] Mitchell AJ, Selmes T. Why don't patients take their medicine? Reasons and solutions in psychiatry. Advances in Psychiatric Treatment 2007; 13: 336-346

[19] Moore DA, Small DA. Error and Bias in Comparative Judgment: On Being Both Better and Worse Than We Think We Are. Journal of Personality and Social Psychology 2007; 92: 972-989

[20] Obrist B. Wie Gesundheit und Resilienz zusammenhängen. UniNova, Wissenschaftsmagazin der Universität Basel, Basel 2012

[21] Russo JE, Shoemaker PJH. Decision Traps: The Ten Barriers to Decision-Making and How to Overcome Them. Broadway Business 1989

[22] Seehausen M, Hänel P. Arzt-Patienten-Kommunikation: Ad härenz im Praxisalltag effektiv fördern. Dtsch Ärztebl 2011; 108 (43): A-2276/B-1918

[23] Smith VL. Rational Choice: The contrast between economics and psychology. Journal of Political Economy 1991; 99: 877-897

[24] Tversky A, Kahneman D. Advances in prospect theory: cumulative representation of uncertainty. In: D Kahneman. Tversky A (Hrsg.): Choices, values and frames. Cambridge: University Press, 2008; 44-66

[25] Weinstein ND. Unrealistic Optimism About Future Life Events. Journal of Personality and Social Psychology 1980; 39: $806-828$

\section{Bibliografie}

DOI https://doi.org/10.1055/a-1228-5634

Nervenheilkunde 2020; 39: 817-823

(c) 2020. Thieme. All rights reserved.

Georg Thieme Verlag KG, Rüdigerstraße 14

70469 Stuttgart, Germany

ISSN 0722-1541 
Punkte sammeln auf CMIE.thieme.de

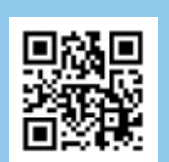

Diese Fortbildungseinheit ist bis zu 12 Monate online für die Teilnahme verfügbar.

Den genauen Einsendeschluss finden Sie beim Modul auf https://cme.thieme.de/CXFGLRA.

Sollten Sie Fragen zur Online-Teilnahme haben, finden Sie unter https://cme.thieme.de/hilfe

eine ausführliche Anleitung. Wir wünschen viel Erfolg beim Beantworten

der Fragen!

Unter https://eref.thieme.de/CXFGLRA oder über den QR-Code kommen Sie

direkt zum Artikel.

VNR 2760512020158723375

\section{Frage 1}

Welches ist keine Voraussetzung für eine rechtwirksame Einwilligung eines Patienten in eine medizinische Behandlung?

A Fähigkeit, die Bedeutung, Tragweite und Risiken der Behandlung zu erfassen.

B Fähigkeit zur eigenen Willensbildung.

C Orientiertheit zu Zeit und Ort.

D Überprüfung der Einwilligungsfähigkeit durch den Arzt.

E Angemessene Aufklärung durch den Arzt.

\section{Frage 2}

Was versteht man nicht unter Non-Adhärenz bei medizinischen Behandlungen?

A Unpünktliche Durchführung der vereinbarten Maßnahmen.

B Mangelndes Überzeugtsein des Patienten von den getroffenen Therapievereinbarungen.

C Unregelmäßige Durchführung der vereinbarten Maßnahmen.

D Änderung der vereinbarten Therapiemaßnahmen durch den Patienten ohne Rücksprache.

E Nichtbefolgung vereinbarter Therapiemaßnahmen.

\section{Frage 3}

Wie häufig kommt es zu Non-Adhärenz bei medizinischen Behandlungen?

A Bei unter $5 \%$ der Behandlungen.

B Bei unter $10 \%$ der Behandlungen.

C Bei etwa $25 \%$ der Behandlungen.

D Bei etwa $50 \%$ der Behandlungen.

E Bei über $50 \%$ der Behandlungen.

\section{Frage 4}

Welche Maßnahme ist bei Non-Adhärenz in Folge von Nachlässigkeit im Allgemeinen nicht wirksam?

\section{A Kritik am Verhalten des Patienten.}

B Stärkung des Selbstvertrauens des Patienten.

C Nicht direktive Erläuterung des Therapieziels.

D Planungshilfen für den Patienten (z. B. Einnahmeprotokolle).

E Einbindung der gewünschten Verhaltensweisen des Patienten an seine Gewohnheiten.

\section{Frage 5}

Welche Maßnahme wird bei Non-Adhärenz in Folge von Labilität empfohlen?

A Betonung der Kompetenz des Arztes.

B Konfrontatives Vorgehen.

C Motivierende Gesprächsführung (Motivational Interviewing).

D Drohung mit Behandlungsabbruch.

E Thematisierung des Urteilsvermögens des Patienten.

\section{Frage 6}

Was versteht man unter dem Dunning-Kruger-Effekt?

A Selbstbezug und Wissensniveau sind positiv korreliert.

B Wer weniger über eine Sache weiß, neigt weniger zur Selbstüberschätzung in diesem Bereich.

C Wer mehr über eine Sache weiß, neigt weniger zur Selbstüberschätzung in diesem Bereich.

D Wer mehr über eine Sache weiß, neigt eher zur Selbstüberschätzung in diesem Bereich.

E Selbstbezug und Wissensniveau sind nicht miteinander korreliert.

\section{Frage 7}

Welcher Persönlichkeitszug bei Patienten führt am ehesten dazu, dass sie dazu neigen, Therapievorschläge abzulehnen?
A Aufgeschlossenheit
B Geduld
C Ungeduld
D Sicherheitsbewusstsein
E Selbstsicherheit

\section{Frage 8}

Was versteht man unter dem klassischen Versicherungsproblem?

A Die Erwartungsbildung des Versicherungsnehmers.

B Die Abwägung zwischen dem Gewinn durch Absicherung und den damit verbundenen Kosten.

C Die Risikobereitschaft des Versicherungsnehmers.

D Die Wahrscheinlichkeit des Schadenseintritts.

E Die Bedeutung des Schadenseintritts in fernerer Zukunft. 


\section{Punkte sammeln auf CME.thieme.de}

Fortsetzung $\ldots$

\section{Frage 9}

Was versteht man unter Overconfidence?

A Übermäßiges Vertrauen in die Kompetenz des Arztes.

$B$ Übermäßiges Vertrauen in einen guten Ausgang der Erkrankung.

C Risikobereitschaft.

D Übermäßiges Selbstvertrauen.

E Übermäßige Gewissheit hinsichtlich der Richtigkeit und Treffsicherheit eigener Urteile.

\section{Frage 10}

Welche Maßnahme ist nicht geeignet, die korrekte Eigenbeurteilung durch den Patienten zu fördern?

A Intensive Patientengespräche.

B Förderung der sachlichen Kompetenz des Patienten.

C Handreichungen und Lesehinweise für den Patienten zum Selbststudium.

D Betonung der Kompetenz des Arztes.

E Zunehmende Komplexität bei der Erläuterung der medizinischen Sachverhalte. 